\title{
Salidroside promotes human periodontal ligament cell proliferation and osteocalcin secretion via ERK1/2 and PI3K/Akt signaling pathways
}

\author{
YUKANG YING and JUN LUO \\ Department of Stomatology, Taizhou Central Hospital, Taizhou, Zhejiang 318000, P.R. China
}

Received October 17, 2016; Accepted December 21, 2017

DOI: $10.3892 /$ etm.2018.6006

\begin{abstract}
Salidroside modulates cell proliferation and serves as an anti-inflammatory and anti-apoptotic agent with efficacy against various diseases. The objective of the present study was to investigate the efficacy of salidroside in enhancing the proliferation of human periodontal ligament cells (hPDLCs). hPDLCs were isolated and the effects of salidroside on cell viability, soluble osteocalcin levels and activation of proliferation-associated signaling pathways were determined using a CCK-8 assay, ELISA and Western blotting, respectively. The results indicated that salidroside induced proliferation of hPDLCs, increased secretion of soluble osteocalcin and enhanced activation of extracellular signal-regulated kinase (ERK)1/2 and phosphoinositide-3 kinase (PI3K)/Akt signaling pathways. These factors were upregulated by salidroside in a dose-dependent manner. The results of the present study suggested that salidroside mediated hPDLC proliferation via the ERK1/2 and PI3K/Akt signaling pathways, as well as osteocalcin secretion. Salidroside may therefore be used as a novel therapeutic agent in the treatment of the tooth-supporting apparatus, progressive tooth destruction or periodontitis.
\end{abstract}

\section{Introduction}

Salidroside is an active constituent and the major active component extracted from the root of Rhodiola rosea $\mathrm{L}$, which has a variety of pharmacological properties (1). Salidroside serves as an anti-inflammatory and anti-apoptotic agent for the treatment of various diseases (2-5).

Salidroside modulates cell proliferation, angiogenic differentiation and apoptosis via signaling pathways including

Correspondence to: Dr Jun Luo, Department of Stomatology, Taizhou Central Hospital, 999 Donghai Avenue, Jiaojiang, Taizhou, Zhejiang 318000, P.R. China

E-mail: junluo_1@126.com

Key words: salidroside, human periodontal ligament cells, proliferation, extracellular signal-regulated kinase $1 / 2$, phosphoinositide-3 kinase/Akt nuclear factor- $\kappa \mathrm{B}(\mathrm{NF}-\kappa \mathrm{B})$, mitogen-activated protein kinases (MAPKs) including extracellular signal-regulated kinase (ERK)1/2 (6), as well as Akt and mammalian target of rapamycin (mTOR) (4,7). For instance, salidroside inhibits the development of lipopolysaccharide (LPS)-induced mastitis in mice by attenuating the LPS-triggered activation of NF- $\mathrm{B}$ and MAPKs (6). Owing to its protective effects against oxidative stress, salidroside has exhibited activity against gastric ulcers (8), hydrogen peroxide-induced apoptosis (3), $\beta$-amyloid-induced cognitive deficits $(9,10)$, bone loss $(1,11)$ and various cancer types, including bladder cancer (12) and lung cancer (13), demonstrating the potential use of salidroside in inhibiting the development of these diseases.

Based on its pharmacological anti-hypoxic and pro-angiogenic effects, salidroside has anti-osteoporotic activity $(1,11)$. Hypoxia or oxidative stress induced osteoblast apoptosis, decreased osteoblast activity, thus leading to a reduction of osteoblastic bone formation and bone loss, which was abrogated by salidroside treatment by enhancing angiogenesis and osteogenesis $(1,11)$. Periodontitis is a chronic multifactorial inflammatory disease characterized by progressive destruction of the tooth supporting apparatus, enhanced cell apoptosis and tooth loss, or rather periodontal bone loss (14-16). To the best of our knowledge, no previous study has assessed the protective effects of salidroside against periodontal bone loss and the mechanism of action.

Since salidroside has protective effect against oxidative stress-associated inhibition of cell proliferation, the present study investigated whether salidroside enhances the proliferation of human periodontal ligament cells (hPDLCs). HPDLCs are the most common cell type in the periodontal membrane and are widely used to investigate processes associated with periodontitis (17). In addition, HPDLCs have stem cell properties and have the capacity to regenerate periodontal ligament tissues $(18,19)$. In the present study, HPDLCs were isolated and treated with salidroside. Cell viability, soluble osteocalcin levels and the expression of proliferation-associated proteins were determined using appropriate methods. Differences between treatments were analyzed to assess the protective effect of salidroside on hPDLC proliferation and the potential of employing salidroside as an effective drug for the treatment of osteoporosis. 


\section{Materials and methods}

Cell line and culture conditions. According to previously published methods (20), hPDLCs were scraped from teeth extracted from 10 young healthy volunteers, aged 14-20 years, with a median age of 18 for orthodontic purposes at the Stomatology Department of Taizhou Central Hospital (Taizhou, China) from July 2015 to December 2015. The study was approved by the Institutional Review Board of Taizhou Central Hospital and informed consent was provided by the patients' guardians (Taizhou, China). Scraped periodontal ligament tissue was minced into small pieces and kept in Dulbecco's modified Eagle's medium (DMEM, Gibco; Thermo Fisher Scientific, Inc., Waltham, MA, USA) containing 10\% fetal bovine serum (FBS; Sigma-Aldrich; Merck KGaA, Darmstadt, Germany) and $1 \%$ penicillin/streptomycin (Invitrogen; Thermo Fisher Scientific, Inc.) at $37^{\circ} \mathrm{C}$ in a humidified incubator with $5 \% \mathrm{CO}_{2}$. All cell extractions were immediately performed and the cells were cultured individually. In all experiments, we used at least 3 cell sources to conduct the tests respectively, and all experiments were conducted in triplicate. But for any 1 comparison showed in the study, only 1 cell source was used. Confluent cells were detached with standard trypsin (Sigma-Aldrich; Merck KGaA) and serially subcultured in fresh DMEM with 10\% FBS and $1 \%$ penicillin/streptomycin under the above conditions. Cells were cultured to the third passage, followed by identification by immunocytochemical staining and use in the experiments of the present study.

Immunocytochemical staining. For the identification of hPDLCs, cells at passage 3 wereimmunocytochemically stained with specific antibodies to vimentin and cytokeratin (21). Cells were fixed with $4 \%$ paraformaldehyde (Biyuntian Co., Shanghai, China) for $30 \mathrm{~min}$, permeabilized with $0.1 \%$ Triton $\mathrm{X}-100$ (Amresco, Solon, OH, USA) for $20 \mathrm{~min}$ and then blocked with $1 \%$ bovine serum albumin (Sigma-Aldrich; Merck KGaA). Next, hPDLCs were incubated with primary antibodies against vimentin (ab8978, 1/1000; Abcam Inc, Cambridge, MA, USA) and cytokeratin (1:200; cat. no. ab9377; Abcam, Cambridge, MA, USA) at $4^{\circ} \mathrm{C}$ for $12 \mathrm{~h}$, and subsequently incubated with horseradish peroxidase (HRP)-conjugated secondary antibody (1:200; cat. no. ab191866; Abcam) for $1 \mathrm{~h}$ at room temperature. DAPI was used for counterstaining to label the nuclei. For visualization of the antibodies, the polymer detection system (PV-9000 kit; Beijing Zhongshan Goldenbridge Biotechnology, Co., Ltd., Beijing, China) with diaminobenzidine (Beijing Zhongshan Goldenbridge Biotechnology, Co., Ltd.) was used according to the manufacturer's instruction.

Cell treatments. Identified hPDLCs at the third passage were cultured in 24-well plates (at a density of $5 \times 10^{3} / \mathrm{ml}$ ) in DMEM with $10 \% \mathrm{FBS}$ at $37^{\circ} \mathrm{C}$ with $5 \% \mathrm{CO}_{2}$. To investigate the influence of salidroside, cells were treated with various concentrations of salidroside $(0,10,20$ and $50 \mu \mathrm{M})$ for $48 \mathrm{~h}$ and cell viability was determined using a Cell Counting kit-8 (CCK-8) assay at time points of $0,6,12,24$ and $48 \mathrm{~h}$. hPDLCs cells were treated with Cell Counting Kit-8 (CCK-8) stain (Sigma-Aldrich; Merck KGaA) for $2 \mathrm{~h}$. The maximum absorbance at $450 \mathrm{~nm}$ was recorded using a microplate spectrophotometer (Bio-Rad
Laboratories, Inc., Hercules, CA, USA). Three individual experiments were performed for each treatment condition and the averaged value was calculated. All results were normalized to optical density values measured from an identically conditioned well that did not contain medium.

ELISA. Soluble osteocalcin levels in the hPDLCs cultured in DMEM were detected using an ELISA kit (Nordic Biosciences Diagnostic, Herlev, Denmark) (22). Three separate experiments were performed for each experimental condition.

Western blotting analysis. Cellular proteins were extracted using radioimmunoprecipitation assay lysis buffer (Solarbio, Beijing, China). Total protein was measured using a micro bicinchoninic acid protein determination kit (Thermo Fisher Scientific Inc.). Cellular protein (20 $\mu \mathrm{g} / \mathrm{lane})$ was then separated by $10 \%$ SDS-PAGE (Solarbio), followed by electro-transfer onto polyvinylidene difluoride membranes (Invitrogen; Thermo Fisher Scientific Inc.). Membranes were then blocked with 5\% non-fat dry milk (BD Biosciences, Franklin Lakes, NJ, USA) in TBS and $0.1 \%$ Tween 20 for $1 \mathrm{~h}$ at room temperature and probed with the following specific primary antibodies supplied by Abcam: Phosphorylated (p)-ERK1/2 (1:1,000; cat. no. ab214362), ERK1/2 (1:1,000; cat. no. ab54230), p-Akt (1:500; cat. no. ab8933), Akt (1:500; cat. no. ab8805), p-phosphoinositide-3 kinase (PI3K) (1:1,000; cat. no. ab182651), PI3K (1:1,000; cat. no. ab86714) and GAPDH (ab9485, 1/2500). Following incubation overnight at $4^{\circ} \mathrm{C}$, cells were probed with the HRP-conjugated secondary antibody (1:1,000; cat. no. ab131368; Abcam) for $60 \mathrm{~min}$ at room temperature. Immunoblot procedures were performed as previously described (23).

Statistical analysis. Values are expressed as the mean \pm standard deviation of three separate experiments. Differences between groups were analyzed using one-way analysis of variance followed by a post-hoc Tukey's test using SPSS software, version 17.0 (SPSS Inc, Chicago, IL, USA). Graphs were drawn using GraphPad Prism 6.0 (GraphPad Software Inc., La Jolla, CA, USA). P $<0.05$ was considered to indicate a statistically significant difference.

\section{Results}

Identification of hPDLCs. For the identification of hPDLCs, immunocytochemical staining for vimentin and keratin was performed. hPDLCs at the third passage stained positively for vimentin (brown; Fig. 1A) and negatively for cytokeratin (counterstained with the nuclear stain DAPI, blue; Fig. 1B). This suggested that the separated hPDLCs came from the mesoderm (23).

Salidroside promotes hPDLC proliferation. Previous studies indicated that salidroside promotes angiogenesis, exerts cytoprotective effects human bone marrow-derived endothelial progenitor cells (BM-EPCs) (7) and blocked the proliferation of cancer cells $(13,24)$, suggesting an anti-cancer effect of salidroside. To investigate the effect of salidroside on the proliferation and viability of hPDLCs, a CCK-8 assay was performed. As indicated in Fig. 2, salidroside induced a significant increase 

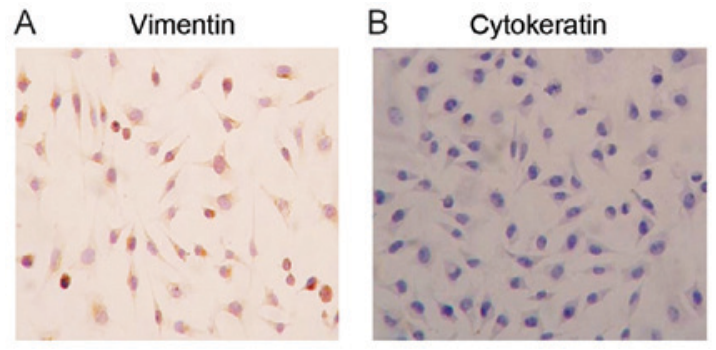

Figure 1. Human periodontal ligament cells at passage three stained (A) positive for vimentin and (B) negative for cytokeratin. Blue indicates DAPI staining of nuclei (magnification x200).

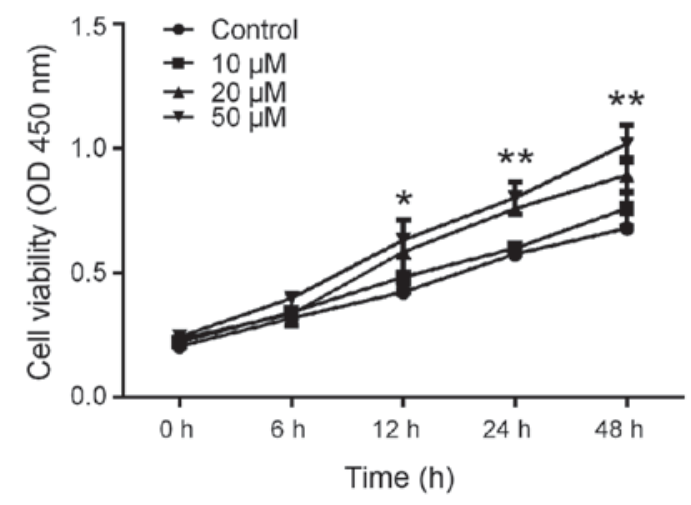

Figure 2. Salidroside promotes hPDLC proliferation. A Cell Counting Kit-8 assay was performed on hPDLCs cells treated with $1,10,20$ and $50 \mu \mathrm{M}$ salidroside for $48 \mathrm{~h}$. All data from three separate experiments were normalized to optical density values measured from an identically conditioned wel that did not contain medium. ${ }^{*} \mathrm{P}<0.05,{ }^{* *} \mathrm{P}<0.01$ vs. control. hPDLCs, human periodontal ligament cells; OD, optical density.

in the amount of viable cells in comparison with those in the control group. In the group treated with salidroside, significant increases in the amount of viable cells compared with those in the control group were noted, confirming that salidroside enhanced hPDLC proliferation.

Salidroside enhances osteocalcin secretion by hPDLCs. To evaluate the influence of salidroside on osteocalcin levels in hPDLCs, the soluble osteocalcin levels in the culture media of hPDLCs were determined using ELISA. HPDLCs were treated with various concentrations of salidroside $(0$, 10,20 and $50 \mu \mathrm{M}$ ) for $24 \mathrm{~h}$ and the culture supernatant was collected for osteocalcin determination. The results indicated a significant increase in osteocalcin levels in the supernatant of salidroside-treated cells, which was dependent on the salidroside concentration (Fig. 3). These results demonstrated that salidroside promoted the secretion of osteocalcin by hPDLCs in vitro.

Salidroside induces activation of ERK1/2 and PI3K/Akt signaling pathways. The ERK1/2 and PI3K/Akt signaling pathways are essential for cell proliferation $(25,26)$. Sustained activation of the ERK1/2 MAPK signaling pathway enhances cell growth (27) and activation of the PI3K/Akt signaling pathway promotes cell proliferation $(26,28)$. Based on this, it was speculated that the enhancement of cell proliferation by salidroside may be associated with the ERK1/2 and PI3K/Akt

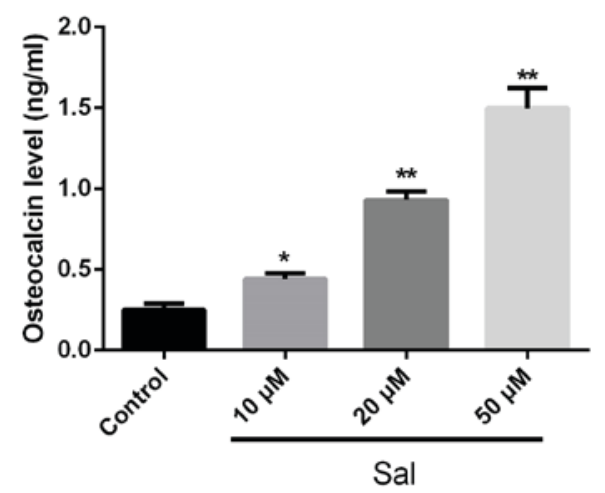

Figure 3. Sal promotes secretion of osteocalcin. Human periodontal ligament cells were treated with $\operatorname{Sal}(0,10,20$ or $50 \mu \mathrm{M})$ for $48 \mathrm{~h}$ and osteocalcin levels were determined using ELISA. ${ }^{*} \mathrm{P}<0.05 ;{ }^{* *} \mathrm{P}<0.01$ vs. control. Sal, salidroside.

signaling pathways. Thus, it was determined whether the expression as well as the activation/phosphorylation status of ERK1/2, PI3K and Akt were upregulated in salidroside-treated cells compared with that of control cells. The results indicated that in cells treated with salidroside, the levels of p-ERK1/2, $\mathrm{p}-\mathrm{PI} 3 \mathrm{~K}$ and $\mathrm{p}$-Akt were increased in a dose-dependent manner as compared with those in the control group (Fig. 4). It was therefore revealed that salidroside enhances the proliferation of hPDLCs through activation of the ERK1/2 and PI3K/Akt signaling pathways.

\section{Discussion}

In the present study, the protective effect of salidroside on hPDLC proliferation was determined. Cells treated with salidroside exhibited an increase in cell viability and secretion of soluble osteocalcin. In addition, the salidroside-mediated proliferation of hPDLCs was associated with activation of the ERK1/2 and PI3K/Akt signaling pathways, which are associated with proliferation.

PI3K and Akt are known to regulate a large variety of critical cellular functions, including proliferation, metabolism, size, survival, migration and angiogenesis (29). ERK is involved in numerous aspects of cell physiology (30) and ERK1/2 signaling is a key factor regulating cell proliferation $(30,31)$. Salidroside treatment was previously reported to promote cell proliferation via several signaling pathways,

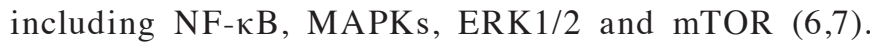
Tang et al (7) demonstrated that treatment of BM-EPCs with salidroside promoted cellular proliferation and angiogenic differentiation. They further demonstrated that the proliferative effect of salidroside on BM-EPCs was associated with phosphorylation/activation of Akt, ERK1/2 and mTOR signaling pathways. The present study confirmed the enhancement of cell proliferation with simultaneous upregulation of $\mathrm{p}$-ERK1/2, p-PI3K and p-Akt protein levels in salidroside-treated hPDLCs, indicating that the cell growth-stimulatory effects of salidroside on hPDLCs are mediated via the ERK1/2 and PI3K/Akt signaling pathways. It was considered that the activation of Akt and ERK1/2 with increased phosphorylation was associated with the promotion of human periodontal ligament cell proliferation $(32,33)$, which was in consistent with the current study. 
A

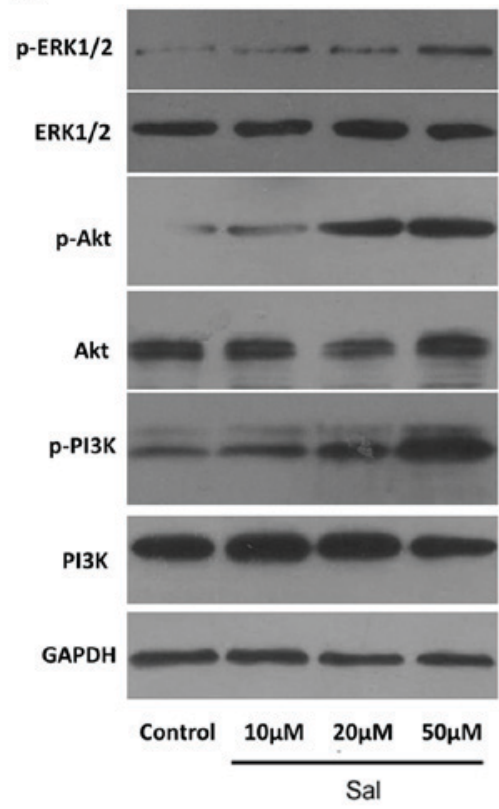

B

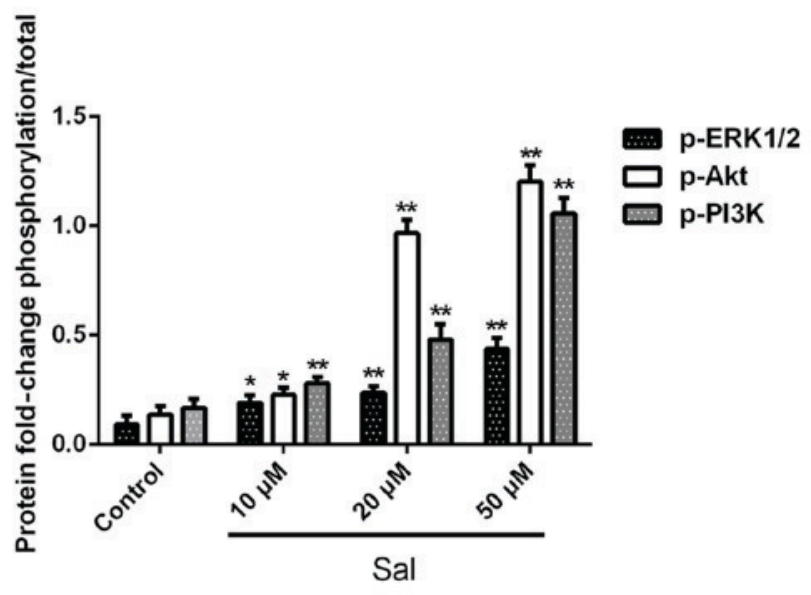

Figure 4. Sal induces activation of ERK1/2 and PI3K/Akt signaling pathways. (A and B) Human periodontal ligament cells were treated with Sal (0, 10, 20 or $50 \mu \mathrm{M})$ for $48 \mathrm{~h}$ and expression levels and phosphorylation status of ERK1/2, PI3K and Akt were determined using western blot analysis. "P<0.05; ${ }^{* *} \mathrm{P}<0.01$ vs. control. Sal, salidroside; ERK, extracellular signal-regulated kinase; PI3K, phosphoinositide-3 kinase.

As previously reported, osteocalcin level are associated with glucose metabolism (34) and $\beta$-cell proliferation $(35,36)$. Specifically, increased osteocalcin promotes $\beta$-cell proliferation, insulin secretion and glucose tolerance (37). Expansion of the $\beta$-cell mass by stimulation of their proliferation is considered to be a potential therapeutic remedy for treating diabetic $\beta$-cell failure (35), suggesting the therapeutic potential of osteocalcin in diabetes. A previous study reported that salidroside enhanced the expression of osteocalcin and reversed $\mathrm{H}_{2} \mathrm{O}_{2}$-induced bone loss in overiectomized rats (1). In addition, the osteocalcin-associated cell proliferation is associated with PI3K/Akt and ERK1/2 signaling pathways $(38,39)$. Osteocalcin is an osteogenic marker (40) and activation of the ERK1/2 signaling pathway induces osteocalcin expression $(39,40)$. Accordingly, in the present study, it was speculated that salidroside treatment induces the secretion of osteocalcin via the PI3K/Akt and ERK1/2 pathways. Indeed, the present results indicated that salidroside promotes the secretion of osteocalcin, as well as the upregulation of PI3K/Akt and ERK1/2 in hPDLCs in a dose-dependent manner, suggesting that supplementation with salidroside may provide a benefit in enhancing bone formation, at least in part via activation of the PI3K/Akt and ERK1/2 signaling pathways. These effects may finally contribute to inhibiting the progressive destruction of the tooth supporting apparatus or the development of periodontitis.

The limitations of the present study include the lack of pre-clinical in vivo data. Furthermore, it is lacking further evaluation of the role of salidroside supplementation on cell apoptosis and other proliferation-associated signaling pathways, including NF- $\kappa \mathrm{B}$ and mTOR $(6,7)$. However, the results of the present study demonstrated the effect of salidroside to enhance the proliferation of hPDLCs.

In conclusion, the present study suggested that salidroside induced the proliferation of hPDLCs. Furthermore, salidroside-mediated cell proliferation was associated with activation of the ERK1/2 and PI3K/Akt signaling pathways, as well as osteocalcin secretion. Based on these results, it may be suggested that salidroside is a potential novel therapeutic agent for the treatment of osteoporosis, progressive destruction of the tooth supporting apparatus and development of periodontitis.

\section{Acknowledgements}

Not applicable.

\section{Funding}

No funding was received.

\section{Availability of data and materials}

All data generated or analyzed during this study are included in this published article.

\section{Authors' contributions}

YY and JL performed all of the experiments and wrote the present study.

\section{Ethics approval and consent to participate}

The study was approved by the Institutional Review Board of Taizhou Central Hospital and informed consent was provided by the patients' guardians (Taizhou, China).

\section{Consent for publication}

Not applicable. 


\section{Competing interests}

The authors declare that they have no competing interests.

\section{References}

1. Zhang JK, Yang L, Meng GL, Yuan Z, Fan J, Li D, Chen JZ, Shi TY, $\mathrm{Hu} \mathrm{HM}$, Wei BY, et al: Protection by salidroside against bone loss via inhibition of oxidative stress and bone-resorbing mediators. PloS One 8: e57251, 2013.

2. Song B, Zhou T, Yang WL, Liu J and Shao LQ: Programmed cell death in periodontitis: Recent advances and future perspectives. Oral Dis 23: 609-619, 2017.

3. Qian EW, Ge DT and Kong SK: Salidroside protects human erythrocytes against hydrogen peroxide-induced apoptosis. J Nat Prod 75 531-537, 2012

4. Kang C, Yuan M, Wang J, Yuan M and Liu C: Salidroside inhibits clinorotation-induced apoptosis in pulmonary microvascular endothelial cells by regulating signaling pathway of endoplasmic reticulum stress. FASEB J (Suppl 1) 30, 2016.

5. Liu S, Yu X,Hu B,Zou Y,Li J, Bo L and Deng X: Salidroside-rescued mice from experimental sepsis through anti-inflammatory and anti-apoptosis effects. J Surg Res 195: 277-283, 2015.

6. Li D, Fu Y, Zhang W, Su G, Liu B, Guo M, Li F, Liang D, Liu Z, Zhang X, et al: Salidroside attenuates inflammatory responses by suppressing nuclear factor- $\mathrm{\kappa B}$ and mitogen activated protein kinases activation in lipopolysaccharide-induced mastitis in mice. Inflamm Res 62: 9-15, 2013

7. Tang Y, Vater C, Jacobi A, Liebers C, Zou X and Stiehler M Salidroside exerts angiogenic and cytoprotective effects on human bone marro-derived endothelial progenitor cells via Akt/mTOR/p70S6K and MAPK signalling pathways. Br J Pharmacol 171: 2440-2456, 2014.

8. Chang X, Luo F, Jiang W, Zhu L, Gao J, He H, Wei T, Gong S and Yan T: Protective activity of salidroside against ethanol-induced gastric ulcer via the MAPK/NF- $\kappa$ B pathway in vivo and in vitro. Int Immunopharmacol 28: 604-615, 2015.

9. Xing S, Yang X, Li W, Bian F, Wu D, Chi J, Xu G, Zhang Y and Jin S: Salidroside stimulates mitochondrial biogenesis and protects against $\mathrm{H}_{2} \mathrm{O}_{2}$-induced endothelial dysfunction. Oxid Med Cell Longev 2014: 904834, 2014.

10. Zhang J, Zhen YF, Pu-Bu-Ci-Ren, Song LG, Kong WN, Shao TM, Li X and Chai XQ: Salidroside attenuates beta amyloid-induced cognitive deficits via modulating oxidative stress and inflammatory mediators in rat hippocampus. Behav Brain Res 244: 70-81, 2013.

11. Li L, Qu Y, Jin X, Guo XQ, Wang Y, Qi L, Yang J, Zhang P and Li LZ: Protective effect of salidroside against bone loss via hypoxia-inducible factor-1 $\alpha$ pathway-induced angiogenesis. Sci Rep 6: 32131, 2016.

12. Liu Z, Li X, Simoneau AR, Jafari M and Zi X: Rhodiola rosea extracts and salidroside decrease the growth of bladder cancer cell lines via inhibition of the mTOR pathway and induction of autophagy. Mol Carcinog 51: 257-267, 2012.

13. Wang J, Li JZ, Lu AX, Zhang KF and Li BJ: Anticancer effect of salidroside on A549 lung cancer cells through inhibition of oxidative stress and phospho-p38 expression. Oncol Lett 7: 1159-1164, 2014.

14. Di Benedetto A, Gigante I, Colucci S and Grano M: Periodontal disease: Linking the primary inflammation to bone loss. Clin Dev Immunol 2013: 503754, 2013.

15. Pacios S, Andriankaja O, Kang J, Alnammary M, Bae J, de Brito Bezerra B, Schreiner H, Fine DH and Graves DT: Bacterial infection increases periodontal bone loss in diabetic rats through enhanced apoptosis. Am J Pathol 183: 1928-1935, 2013.

16. Tonetti MS and Van Dyke TE; Working group 1 of the joint EFP/AAP workshop: Periodontitis and atherosclerotic cardiovascular disease: Consensus report of the Joint EFP/AAP workshop on periodontitis and systemic diseases. J Periodontol 84 (4 Suppl): S24-S29 2013.

17. Lee SI, Yi JK, Bae WJ, Lee S, Cha HJ and Kim EC: Thymosin beta-4 suppresses osteoclastic differentiation and inflammatory responses in human periodontal ligament cells. Plos One 11: e0146708, 2016.

18. Hasegawa M, Yamato M, Kikuchi A, Okano T and Ishikawa I Human periodontal ligament cell sheets can regenerate periodontal ligament tissue in an athymic rat model. Tissue Eng 11: 469-478, 2005.

19. Nagatomo K, Komaki M, Sekiya I, Sakaguchi Y, Noguchi K, Oda S, Muneta T and Ishikawa I: Stem cell properties of human periodontal ligament cells. J Periodontal Res 41: 303-310, 2006.
20. Han J, Meng HX, Tang JM, Li SL, Tang Y and Chen ZB: The effect of different platelet-rich plasma concentrations on proliferation and differentiation of human periodontal ligament cells in vitro. Cell Prolif 40: 241-252, 2007

21. Xia L, Zhang Z, Chen L, Zhang W, Zeng D, Zhang X, Chang J and Jiang X: Proliferation and osteogenic differentiation of human periodontal ligament cells on akermanite and $\beta$-TCP bioceramics. Eur Cell Mater 22: 68-83, 2011.

22. Giuliani N, Morandi F, Tagliaferri S, Lazzaretti M, Bonomini S, Crugnola M, Mancini C, Martella E, Ferrari L, Tabilio A and Rizzoli V: The proteasome inhibitor bortezomib affects osteoblast differentiation in vitro and in vivo in multiple myeloma patients. Blood 110: 334-338, 2007.

23. Fu X, Tian J, Zhang L, Chen Y and Hao Q: Involvement of microRNA-93, a new regulator of PTEN/Akt signaling pathway, in regulation of chemotherapeutic drug cisplatin chemosensitivity in ovarian cancer cells. FEBS Lett 586: 1279-1286, 2012.

24. Lv C, Huang Y, Liu ZX, Yu D and Bai ZM: Salidroside reduces renal cell carcinoma proliferation by inhibiting JAK2/STAT3 signaling. Cancer Biomark 17: 41-47, 2016.

25. Dai J, Peng L, Fan K, Wang H, Wei R, Ji G, Cai J, Lu B, Li B, Zhang $\mathrm{D}$, et al: Osteopontin induces angiogenesis through activation of PI3K/AKT and ERK1/2 in endothelial cells. Oncogene 28: 3412-3422, 2009

26. Waseem T, Duxbury M, Ashley SW and Robinson MK: Ghrelin promotes intestinal epithelial cell proliferation through PI3K/Akt pathway and EGFR trans-activation both converging to ERK 1/2 phosphorylation. Peptides 52: 113-121, 2014.

27. Ishii A, Furusho M and Bansal R: Sustained activation of ERK1/2 MAPK in oligodendrocytes and schwann cells enhances myelin growth and stimulates oligodendrocyte progenitor expansion. J Neurosci 33: 175-186, 2013.

28. Yang N, Hui L, Wang Y, Yang H and Jiang X: SOX2 promotes the migration and invasion of laryngeal cancer cells by induction of MMP-2 via the PI3K/Akt/mTOR pathway. Oncol Rep 31: 2651-2659, 2014.

29. Serra V, Scaltriti M, Prudkin L, Eichhorn PJ, Ibrahim YH, Chandarlapaty S, Markman B, Rodriguez O, Guzman M, Rodriguez S, et al: PI3K inhibition results in enhanced HER signaling and acquired ERK dependency in HER2-overexpressing breast cancer. Oncogene 30: 2547-2557, 2011.

30. Martin P and Pognonec P: ERK and cell death: Cadmium toxicity, sustained ERK activation and cell death. FEBS J 277: 39-46, 2010.

31. Roux PP and Blenis J: ERK and p38 MAPK-activated protein kinases: A family of protein kinases with diverse biological functions. Microbiol Mol Biol Rev 68: 320-344, 2004.

32. An S, Huang X, Gao Y, Ling J, Huang Y and Xiao Y: FGF-2 induces the proliferation of human periodontal ligament cells and modulates their osteoblastic phenotype by affecting Runx 2 expression in the presence and absence of osteogenic inducers. Int J Mol Med 36: 705-711, 2015

33. Padial-Molina M, Volk SL and Rios HF: Periostin increases migration and proliferation of human periodontal ligament fibroblasts challenged by tumor necrosis factor- $\alpha$ and Porphyromonas gingivalis lipopolysaccharides. J Periodontal Res 49: 405-414, 2014.

34. Booth SL, Centi A, Smith SR and Gundberg C: The role of osteocalcin in human glucose metabolism: Marker or mediator? Nat Rev Endocrinol 9: 43-55, 2013.

35. Wei J, Hanna T, Suda N, Karsenty G and Ducy P: Osteocalcin promotes $\beta$-cell proliferation during development and adulthood through Gprc6a. Diabetes 63: 1021-1031, 2014.

36. Chang D, Xu H, Guo Y, Jiang X, Liu Y, Li K, Pan C, Yuan M, Wang J, Li T and Liu C: Simulated microgravity alters the metastatic potential of a human lung adenocarcinoma cell line. In Vitro Cell Dev Biol Anim 49: 170-177, 2013

37. Kaw A, Fraga D and Fischer C: Effect of osteocalcin on human beta cell proliferation and function and its role on insulin secretion. Pancreatol 13 (Suppl): S64, 2013.

38. Gerard K and Ducy PF: Undercarboxylated/uncarboxylated osteocalcin increases beta-cell proliferation, insulin secretion, insulin sensitivity, glucose tolerance and decreases fat mass. Patent AU2007294732. Filed September 13, 2007; issued April 23, 2009.

39. Liu J and Yang J: Uncarboxylated osteocalcin inhibits high glucose-induced ROS production and stimulates osteoblastic differentiation by preventing the activation of PI3K/Akt in MC3T3-E1 cells. Int J Mol Med 37: 173-181, 2016.

40. Han Y, Xu G, Zhang J, Yan M, Li X, Ma B, Jun L, Wang SJ and Tan J: Leptin induces osteocalcin expression in ATDC5 cells through activation of the MAPK-ERK1/2 signaling pathway. Oncotarget 7: 64021-64029, 2016. 